\title{
Virtual Prototyping Using Graphical Simulation and Advanced Programming Techniques
}

\author{
K.-D. Thoben, U.Berger, J. Bauer, A. Schmidt \\ BIBA - Bremen Institute for Industrial Technology and \\ Applied Work Science at the University of Bremen \\ P. O. Box 3305 60, D-28335 Bremen, Germany \\ email:as@biba.uni-bremen.de
}

\begin{abstract}
This paper presents a practical approach towards Virtual Prototyping. The fields of application are One-of-a-Kind products and industrial prototypes. The first example is taken from the problem area of typically high complex capital goods being virtually modelled and simulated prior to the real setting-up. The strategy of complimentarily developing both, the prototype's mechanics and its functionality and the issues of concurrent and distributed team-work are characterised. Enabling technologies for the iterative setting-up of the real prototype are described with reference to practical experience. The second example is the virtual and the touchable prototyping of sheet metal parts, incorporating the FEM simulation of the process as well as the closed prototyping chain for a fast verification of the results. An enabling technology is the triangulation of parametric CAD surface models, a technique to represent random solid bodies as a polyedrical mesh, from which "real" and "virtual" prototypers will equally profit. The possibility to include surface data acquired by 3D measurement systems is also discussed. As a conclusion, the modelling of process chains is seen as an adequate approach to achieve better clarity in production as well as in the product development, describing actions and streams in between them. Their addition leads to a reference model for rapid product development and One-of-a-Kind Production, providing an abstract view of the various process chains in a neutral and logical manner. This is a necessity to reach the aim of production in the end of this century: "make just what is needed when it is needed".
\end{abstract}

\section{Keywords:}

Virtual Prototyping, graphical simulation, industrial robots, car disassembling, Neural Network, off-line programming, optical 3D-measurement, CAD surface model, sheet metal forming, Rapid Prototyping, Concurrent Engineering, triangulation, Stereolithography STLfile, Reverse Engineering, rendering 


\section{INTRODUCTION}

The competitiveness of industrial countries depends on their ability to anticipate customer needs, respond quickly to the needs they can articulate and develop new, unique products. According to actual market data, the "time-to-market" phase requires ever larger investments of time and money for product development. With relatively high production costs, getting to market first is the key to profitability in the short term.

Rapidly developing and manufacturing according to customer wishes, so-called on-demand manufacturing, is the challenge. The time has gone when products can be developed first and then offered to a market or a potential customer. The implications for manufacturing systems design are profound. Therefore, there is a need to develop the capability, both technically and organisationally, to rapidly transform new ideas into prototypes (first-of-a-kind). Additionally related manufacturing equipment has to be developed, maintained and enlarged. As a consequence, advances in information and communication technologies and related organisational approaches have to make the required breakthroughs possible.

Besides other approaches, the computer-based visualisation of an early version of a product, parallel to the possibility of analysing, testing and manipulating this non-physical representation in a computer-generated virtual environment, plays an important role in achieving a shorter "time-to-market". As the basic principle of Virtual Prototyping is the early consideration and integration of entire life-cycle related requirements and the resulting early prediction and evaluation of the product behaviour, various approaches are possible. These areas of application range from simple visualisation to attract a customer by a simple but early virtual version of the product requested (i.e. product visualisations during tendering) to most complex FEM applications analysing the strength, functionality, and manufacturing and product behaviour during operation.

As, in principle, the idea of Virtual Prototyping is not really new, new hardware and software techniques and technologies can make Virtual Prototyping an excellent extension to existing approaches like Conjoint Measurement, DfX (Design for Manufacturing, Design for Assembly, etc.), Feature-Based Design, NC simulation, etc. and Virtual Prototyping can integrate and make best use of these single-minded integration approaches. In addition to this, further benefits of Virtual Prototyping can be achieved by a dynamic and iterative integration of the real and the virtual world and by an integration of the various views of different partners during a globally distributed product development.

In the following, experiences gained in different projects applying various approaches of Virtual Prototyping and related enabling technologies for speeding up product development of capital and consumer goods will be described. In a next step these approaches and enabling technologies will be characterised and classified to define a systematic way for improving product development and production through Virtual Prototyping.

\section{PROTOTYPING OF FREE-FORMED SHEET METAL PARTS}

Regarding the product development cycle itself, the basic difference lies between the still widely used sequential way of defining a new product, and the more advanced methods with a consequent parallelism and a well-organised iterative approach. These methods are often referred to as Concurrent Engineering, Simultaneous Engineering and Agile Engineering, and they suggest the following rules to product developers:

- Gather any information needed in the starting phase. Begin with a large interdisciplinary expert team; decrease number of people while refining the solution. 
- Make changes early, when this is still inexpensive.

- Make decisions early. Test out a large variety of solutions and select the best at the beginning instead of repairing mistakes resulting in large expenses at the end.

- Use communication tools (CSCW).

Basically, Concurrent Engineering can be performed with conventional means like drawing boards up to a limited complexity. But for planning complex products like aircraft, ships and manufacturing plants, more advanced design and communication tools - CAD, CAM, CAE, RP and EDM Systems - are highly recommended.

At first will be given an insight into potentials and demands observed with computational geometry manipulation and RP Techniques. Then, these consequences will be extended towards Virtual Prototyping.

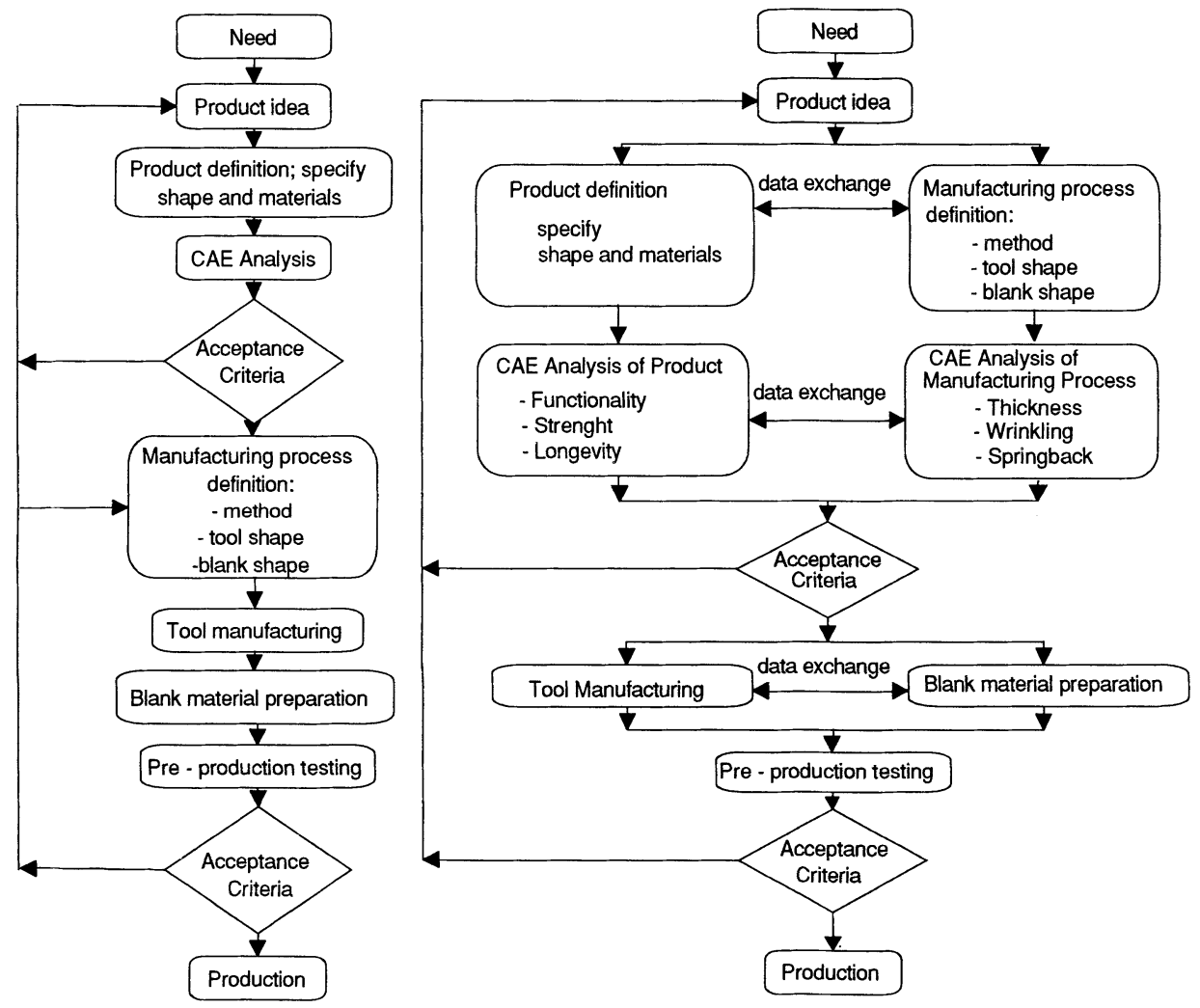

Figure 1 Schematic process chain: left with conventional method, right with integrated process chain

With Rapid Prototyping Technologies, models with good surface quality and accuracy can be built today. However it is not yet possible to produce a sheet metal forming tool directly by Rapid Prototyping. The potential of the new manufacturing techniques can only successfully be used by a sequence of process steps incorporating RP technologies. This sequence consists of: 
- Techniques of triangulating trimmed surfaces in parametric space.

The standard data exchange format in Rapid Prototyping is STL. It incorporates a primitive polyhedrical representation of three dimensional bodies. This has the advantage of simplicity and wide application range, but the disadvantages are limited accuracy, large file sizes and comparatively difficult data preparation in the case of converting surface CAD-models to STL - format.

- Reverse Engineering

Quick modelmaking like RPT supply a powerful "downstream" path, from the abstract geometry data towards the physical representation of a part. "Reverse Engineering" tools are supposed to do just the opposite: with 3D measurement systems, a geometrical shape aquisition is performed first. Then, an abstract description of the object is reconstructed with the aid of dedicated software tools. The result of this is a valid CAD model of the part, even if it was comletely unknown before.

- Rapid Prototyping Technology (in this case Stereolithography) is used to produce a mould.

With so-called Rapid Prototyping Techniques (synonyms: Layer Manufacturing, Solid Freeform Manufacturing, ...), parts with nearly unlimited geometries can be built automatically, with fully automated path preparation. On the other side, the material properties and the accuracy of the built parts are within fixed limits.

- Casting technique using a high strength concrete material.

With methods like plaster moulding, vacuum casting and investment casting, moulds, tools and functional prototypes can be derived from the RP part directly. The casting materials palette can cover a wide range of material properties, e.g. high strength and high wear resistance in the case of moulds.

The main idea behind a new process chain is to build forming tools by using easily castable amorphous material and integrate the Stereolithography process for fabrication of the casting shell (negative geometry). To attain these goals, an information transfer process was developed from a CAD surface model of the formed part by means of Rapid Prototyping Technologies towards an advanced sheet metal forming process [3, 4].

The cast material consists of a mineral binding material with addition of ultra fine particles, which are typically 100 times smaller than the cement particles. These small particles, combined with a dispersing agent make up an extremely dense packing which results in a very high strength as well as the ability anchor to particles and fibres in the matrix [5]. It is a cheap material which is easy to handle, with no environmental risks connected to its preparation and disposal [6].

- Application of a forming operation which is specially suitable to produce small batches. Tool manufacturing is very expensive and ineconomical for small volume production. For sheet metal forming, methods with "effecting means" are available, without the necessity of the male and the female die. These are rubber pad forming, fluid cell forming and pressure forming.

The rubber-pad-forming technology is established for flexible and cheap forming operations. It uses a special type of rubber-die pressing in which the rubber die is totally enclosed, thereby exerting fluid pressure on the workpiece. The forming pressure is up to 1000 bar.

The main advantage of this principle is the possibility to form a part with only one die, where normally an upper and lower die are necessary.

The steps for manufacturing were:

- Production of blanks with a punching/nibbling machine after the CAD data had been postprocessed.

- Applying the rubber pad forming process to the blanks.

Figure 1 shows the conventional method and the integrated method for process chains for prototype parts. 


\subsection{Integrated Process Chain for Sheet Metal Parts: a Case Study}

As an example, a prototype protection cap was realised for a position lamp (shipbuilding industry). The material was stainless steel (wear and corrosion resistant) and the required thickness of the sheet metal was $0,7 \mathrm{~mm}$. (Figure 2)

Both a computer-aided optimisation of the forming process (finite-element methods) as well as close co-operation with tool manufacturing experts are necessary; otherwise some undesired results in the manufacturing process could occur, such as wrinkles; spring-back effects, cracks or distortions.
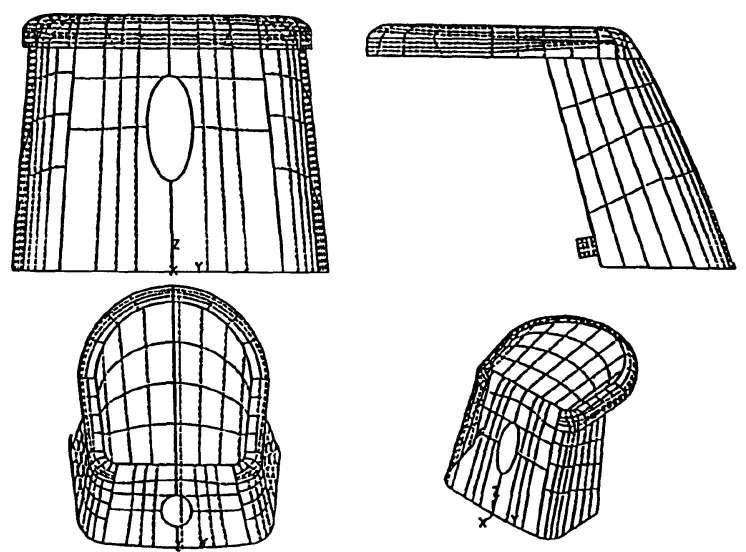

Figure 2 CAD surface model of protection cap

Derived from the product's CAD data, counterparts for the tool construction were designed. After triangulation and slicing on computer, these casting shells were fabricated on a Stereolithography machine. Then, the casting was performed with a special kind of ultra-fine concrete.

Parallel to the tool manufacturing, the preparation of the blank material was started. Based on the workpiece and tool data, the geometrical design of the sheet metal was done on a 2DCAD/CAM system.

Now the metal part production started: the blanks were manufactured on a CNC-controlled punching- and nibbling - machine, and the forming operation was performed on a rubber pad forming press.

\subsection{Triangulation of Trimmed Surfaces in Parametric Space}

An essential key to automated manufacturing processes is the manufacturing data preparation, after the CAD model of a product or a tool is available. On the field of data-pre-processing for Stereolithography, a software package was written that is capable to triangulate parametric surfaces to a polyedrical representation (Stereolithography STL-file). It is able to find faults in STL-files and repairs them automatically, can handle layer information and create STL out of it again, and has visualisation and graphical user interface. For "closing the loop" from CAD 
model to physical part and back again, it will be of interest to integrate 3D optical sensors with the aim of doing Reverse Engineering, too.

The faceting of parametric surfaces is of great interest for many CAD/CAM applications. After the faceting, a surface model can be displayed with a nearly natural appearance in realtime. The faceting technique is also applied to other numerical calculations for surface models.

The authors of [1] were particularly motivated by the task of writing an interface between CAD systems and a Stereolithography apparatus (SLA). The current interface to an SLA requires that a part to be machined must be triangulated. Since a triangulation for an SLA must correspond to a real object, no cracks and improper intersections are allowed in the model. Further, triangles must meet along with common edges, and triangle normals must point away from the object. These requirements are easily met by a solid model. In practice, however, the sophisticated models found in the automotive and aerospace industry consist of free-form surfaces. This makes the triangulation of the models much more difficult, especially if trimmed surfaces are handled.

Unfortunately, most of the known algorithms are not designed to process a trimmed surface. An extension of an existing triangulation algorithm performs triangulation for trimmed surfaces completely in parametric space. Strategies for avoiding cracks between patches and surfaces are also implemented.

To design freeform surfaces with nearly random shape, three mathematical methods are applied: first, the whole surface is dissected to smaller pieces (called "patches"); each of these is described by a polynom with limited degree. Second, the polynoms are not described explicitly as $\mathrm{s}=\mathrm{f}(\mathrm{x}, \mathrm{y}, \mathrm{z})$ in euclidean space, but as functions $\mathrm{s}=\mathrm{f}(u, v)$ with $u, v$ being parameter values in a "curved" coordinate system - easily describable as "run length" of the surface in the two different directions $u$ and $v$. Third, undesired portions of the so-defined freeform surface can be cut off by "trim curves". These are defined in the same parameter coordinate system as the surface patches themselves.

So, a "piecewise polynomial surface is a grid of parametric polynomial patches which can be considered as a tensor product of two parametric variables, $u$ and $v^{\prime \prime}$. The basic principle of the following triangulation procedure is, to perform it in the parameter coordinate system $u, v$ instead of the euclidean space $x, y, z$. The advantage is, that thet a considerable number of algorithms now
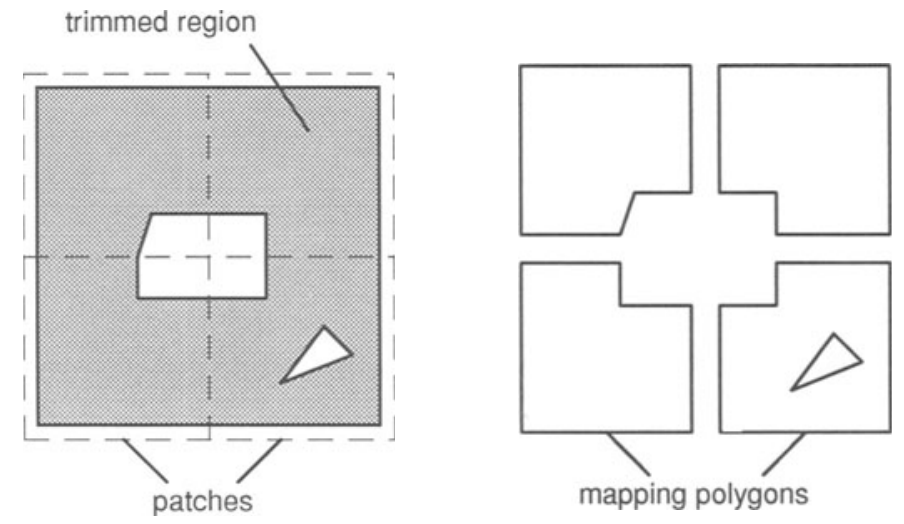

Figure 3 Generation of mapping polygons by splitting of trimmed regions. 
By splitting the surface into its patches again, the parameter space is subdivided into a set of polygons, called mapping polygons. Each of these polygons thus corresponds to a patch on the surface. To generate the mapping polygons in parametric space, the splitting and refinement procedures can be efficiently performed using the Euler operator [8]. After splitting, the boundary of a mapping polygon consists of splitting lines and trimming curves (Figure 3). By checking the neighbourhood information for pairs of half-edges, one can easily detect cracks.

Avoiding cracks between surfaces is more difficult. This can be done by calculating the shortest distance between two line segments (edges), since the trimming curves have already been approximated by line segments. If two trimming curves match, they are geometrically merged assigning the same coordinates to the matching points on the curves, to prevent cracks and improper intersections between surfaces.

To ensure that the model resulting from the merging is manufacturable by the SLA, it is required that the model satisfies the manifold criteria [2] as follows:

- Every edge is shared exactly by two faces.

- Every vertex is surrounded by a single cycle of edges and faces.

- Faces may not intersect each other except at common edges and vertices.

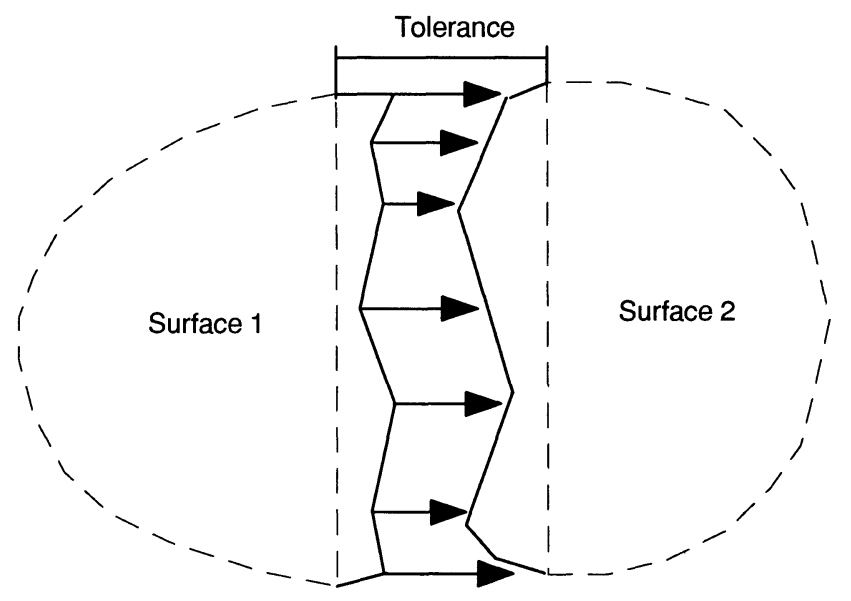

Figure 4 Merging of boundary curves of two matching surfaces

On the basis of these criteria, each edge on the boundaries can be merged only once. The merging procedure expands by following matching edges in both directions until the end points of edges are reached (there, naturally, no further match can occur). Finally, two pairs of end points of merging edges are assigned to the same co-ordinates in Euclidean space.

Merging boundaries of neighbouring surfaces cause some artefacts. However, these modifications are all within usually small, specified tolerances. On the other hand, the modifications are inevitable to make a surface model manufacturable for SLA machines at all (Figure 4). 
Although the algorithm has been implemented as an interface for SLA, it is expected that it would also be suitable for the rendering procedures needed for realistic image generation as needed in Virtual Reality applications.
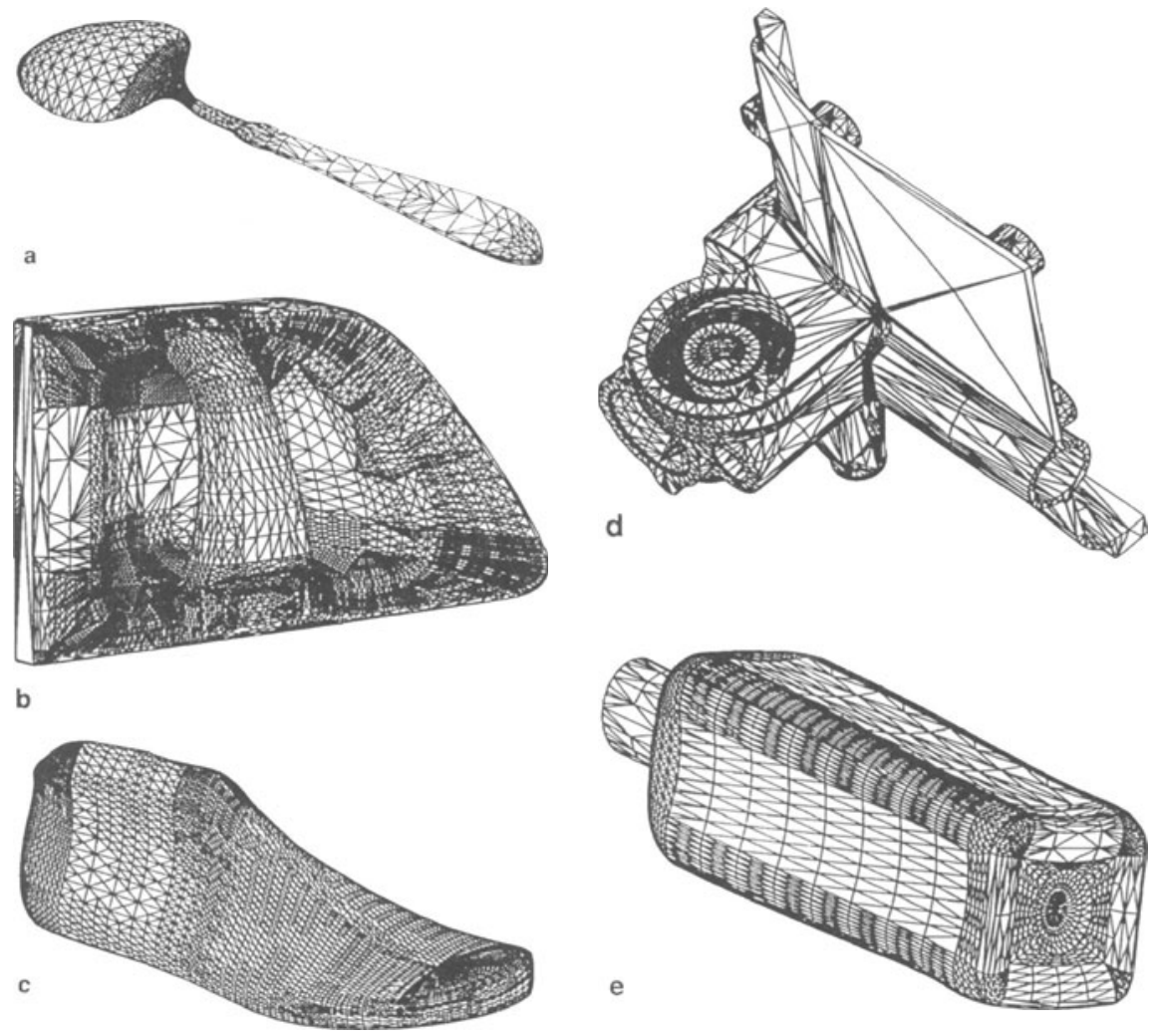

Figure 5 Triangulation models created by the described algorithms; (a) spoon, (b) door handle, (c) shoetree ${ }^{I}$, (d) engine part ${ }^{2}$, (e) liquor bottle ${ }^{3}$

\subsection{Integrating sensor data in a polygonal shape representation}

For "Reverse Engineering" on the basis of 3D sensor data, some very similar problems occur as described before. One major problem is again to meet the manifold criteria. Presently the STL interface software will be extended to make it capable to create a mesh out of the sensor data and sweep it to a solid model. The regions that are "behind" the measured points, representing the parts of the object that cannot be seen by the sensor, are represented by solid "sticks", stretching out behind the triangle mesh to infinity. Then, such "solid views" are intersected, generating an STL representation of the whole object once all views are performed. The advantage of this procedure is supposed to be that it will not need a CAD system to do a shaded visualisation or direct replication via Rapid Prototyping.

\footnotetext{
${ }^{1}$ Courtesy of KF Desma, Achim, FRG

2 Courtesy of Porsche AG, Weissach, FRG

${ }^{3}$ Courtesy of Cisigraph GmbH, FRG
} 


\subsection{Results}

The described approach chain can be consequently supported by Concurrent Engineering Methods and Layer Manufacturing Technologies. Tremendous time reduction was realised. The experiments show that the prototype could be manufactured with this process. Currently, coating the dies by wear-resistant surface materials (nickel, chromium) is under research.

Software tools using a triangular representation of freeformed bodies proved to supply a good means for supporting early phases of production and product development, where only rough data is available. A software package initially dedicated to support process planning of Rapid Prototyping facilities shows to be a basis that can easily be expanded towards follow-up processes like casting moulds, FEM simulation, 3D sensor data integration, and many more.

The described process chain allows for cheap and fast production of an experimental sheet metal forming tool. Thus the test phase of the tool can start at an early stage of the product development. Because one starts from a CAD model and uses a Rapid Prototyping Technology, the tool can be changed quickly and cheaply. Several tests can be made without violating time frame and budget. A final steel tool can be produced starting from the same data. The possibility of tests using an experimental tool results in a greater safety concerning functionality of the tool and quality of the part. Designer and toolmaker both can create an optimal product and tool design easier.

\section{VIRTUAL PROTOTYPING OF A ROBOT CELL FOR DISASSEMBLING}

The prototype development of a robot cell for disassembling of car components is an example that represents One-of-a-Kind capital products. It demonstrates that the physical complexity of the One-of-a-Kind product often corresponds to its functional complexity. Because the functional concept the mechanical structure have a strong impact on each other, both sides have to be tackled at the same time. For the development of solutions that have a high functional and geometric complexity the concepts of Virtual Prototyping offer effective support.

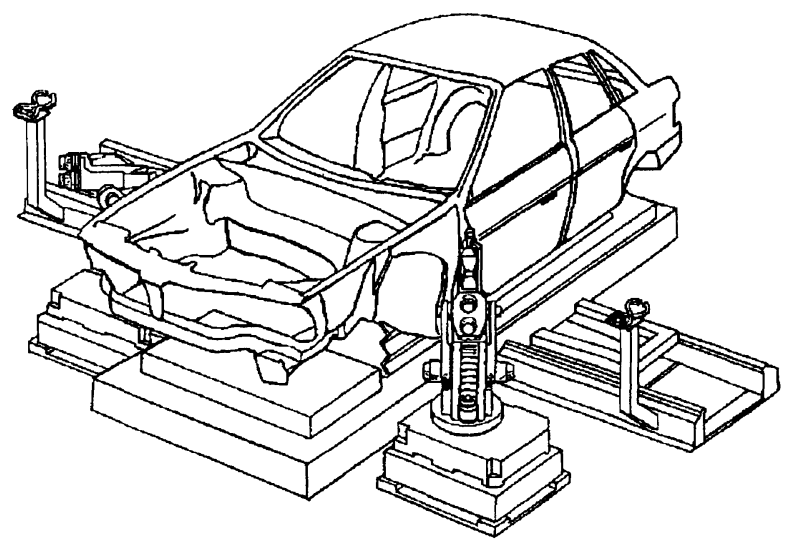

Figure 6 Principle of the NEUROBOT robot cell for car disassembling 
Often experts from different companies are involved in the development. Then, such projects are, in fact, distributed over different sites. This means that the project has to be carried out by distributed specialist groups working concurrently in close contact with each other. Consequently, the technical and organisational concepts of Virtual Prototyping and Concurrent Engineering have to be merged by enabling technologies to support the development of such One-of-a-Kind capital products like this disassembling cell.

The NEUROBOT ${ }^{4}$ prototype cell is designed to be a self-consistent, error tolerant and autonomous intelligent robot and multi-sensor system based on Neural Network and Adaptive Control concepts. It will be able to perform disassembling operations in non-structured environments and in the absence of design and manufacturing descriptions of the car components (Figure 6). Besides the fact that the recycling of old cars is a problem of increasing relevance there are a number of technical challenges that motivate the work on NEUROBOT. The project will demonstrate the economic and technological potential for the automation of disassembling tasks of a large variety of car components.

The mechanic and sensor structures of the cell have to have a high degree of problem adjustability and kinematic freedom to reach the highest possible flexibility for the effective disassembling of as many as possible components with a large variety. The system has to acquire autonomically the information necessary for the disassembling process. The required task sequences need to be planned for each individual disassembling problem by the system itself based on the information acquired before. The programs for the robot cell must be generated automatically considering reachability and collision avoidance.

\subsection{Complementary Virtual Prototyping of the Mechanic and Functional Components}

While many fields of advanced technology are touched within this project and because of their strong cross relations, the mechanical design, the development of the sensor and control systems as well as their programs is carried out concurrently by different expert teams. For these purposes a computer environment with powerful simulation functionality for the development of the Virtual Prototype is established.

1. The structure of the car disassembling robot system is described and the interfaces for the information exchange between the system components itself and between the virtual and the real environment aire defined (Figure 7).

2. The development tools for the Neural Networks in the NEUROBOT controller and sensor systems are chosen.

3. The simulation environment for the development of the virtual prototype is established by joining IGRIP with the controller development tools.

Because of the complexity of the disassembly task various different approaches for the generic car model, the generic disassembling plan, the network architecture, the learning rules, etc. are assessed and the performance of each approach is evaluated. The performance of the whole system will be tested frequently to avoid cost-intensive changes at a late time. Thus, the development phase is an iterative process, distributed between a number of concurrently working project partners.

As the Virtual Prototyping environment has interfaces to the real world (e.g. translators for different robot control languages or sensor ports) the real system components can be tested

\footnotetext{
${ }^{4}$ The development is carried out in the context of the ESPRIT project no. 8338 "NEUROBOT - Neural Network Based Robots for Disassembling and Recycling of Automotive Products" funded by the Commission of the European Communities.
} 
with the virtual system components while these components haven't been set up or while these components are at another project partner's development site.

The function of the system in different situations can be tested with the real sensor system connected to the virtual model. Or in turn, the real robot can be tested in connection with the virtual controller, and so forth. This allows the test of the entire system at any stage of the work so that the soundness of the development can be assessed frequently.

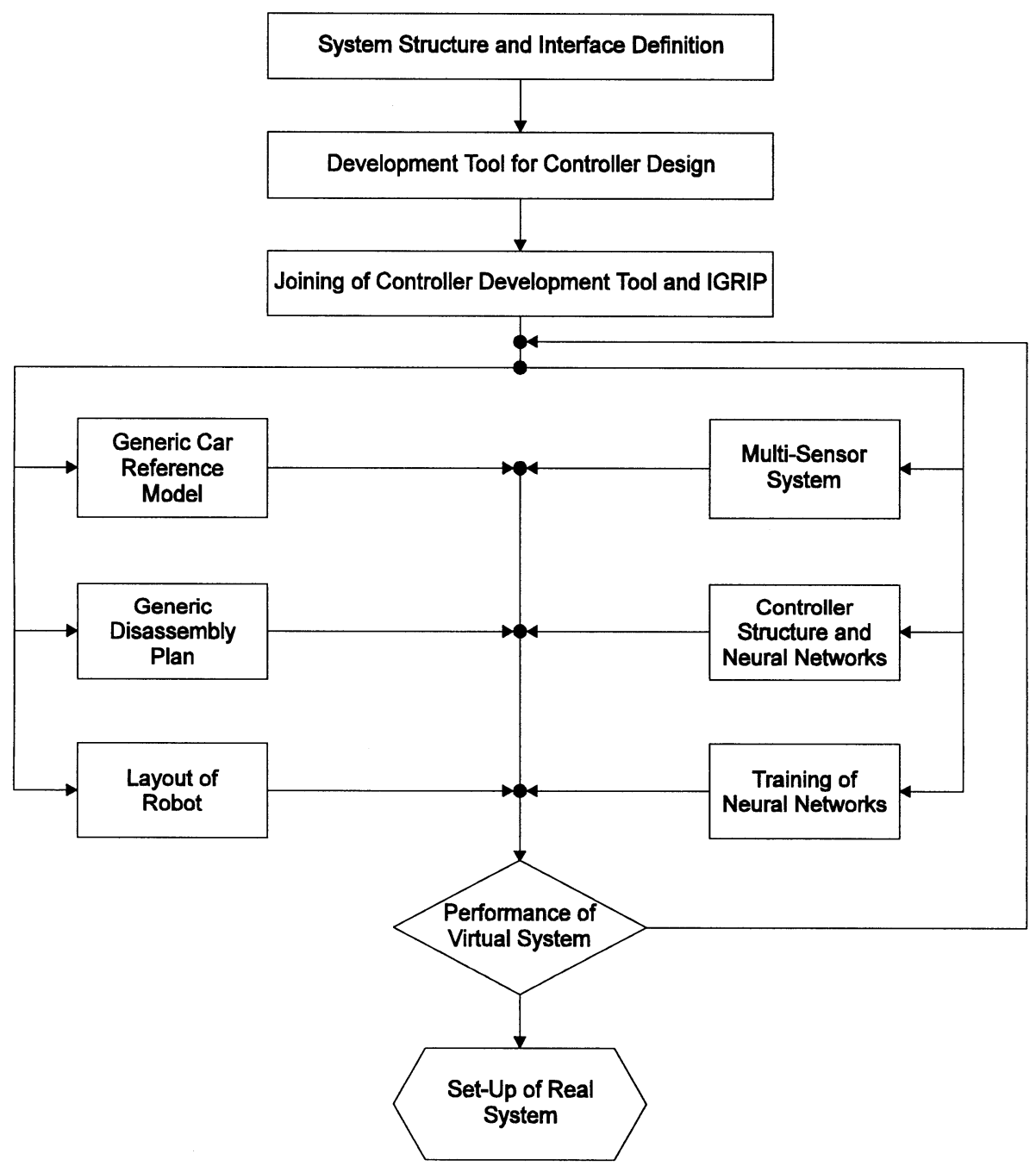

Figure 7 Iterative structure for the Complementary Virtual Prototyping of NEUROBOT. 


\subsection{Characteristics of the Complementary Virtual Prototyping}

The development of One-of-a-Kind capital goods using approaches of concurrent, distributed complimentary Virtual Prototyping has the following characteristic features:

- changes, new additions or different options are easily included,

- results and cross impacts of different approaches become apparent immediately,

- functional concept and mechanical structure are developed complimentarily,

- interfaces to the real world from many points within the virtual prototype,

- real components are tested together with virtual components,

- opportunity for the assessment of the entire system at any stage of the setting-up,

Thereby, the described method has the following advantages over the traditional way of development:

- opportunity for distributed and concurrent development,

- conceptual weaknesses are rectified in the virtual prototype,

- the soundness of the development can be assessed frequently,

- errors are easier to identify,

- functionality and mechanical structure are consistent,

- test bed representing the entire system is available during the whole setting-up

- expenditures for changes in the virtual prototype are far less than for changes in the real prototype

- work is more efficient,

- prototype is developed faster,

- prototype with a higher quality.

\subsection{Linking Graphic Simulation with Development Tool for Intelligent Controllers}

The development of this prototype robot cell is carried out in a virtual simulation environment. This environment consists of the powerful graphic simulation and robot off-line-programming tool IGRIP of DENEB Robotics and development tools for Neural Networks and Artificial Intelligence applications. The user interface comprises of many tools from a CAD and layout environment as well as simulation, translation and communication tools (Figures 8 and 9).

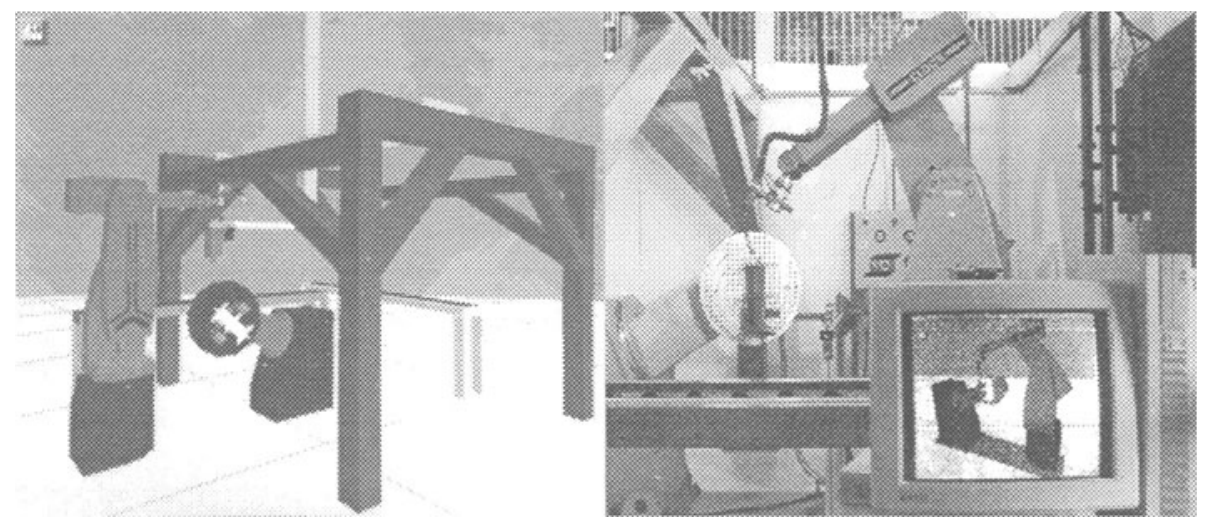

Figure 8 Screen from the IGRIP off-line programming tool

Figure 9 Robot cell and its simulation 
The off-line-model of the disassembling cell will be linked via socket communication with the Neural Network development tool. Through this communication link, simulated sensor signals, graphic and geometric information and control data are exchanged. This facilitates the generation of realistic training data for the Neural Networks. The connection to the robot 32 bit controller is established via IGRIP up- and download. The measurement PC and IGRIP can exchange data via TCP/IP and NFS.

\subsection{Linking Graphic Simulation with 3D-Sensor and Industrial Robot}

With an optical 3D-sensoric system on an industrial robot linked with a CAD/CAM environment it is possible

1. to carry out the work piece handling and the manufacturing sequence in a virtual production scenario to avoid program faults, collision, exceeding the work range or payload limit during the real robot action [7] and

2. the product tolerances can be compensated through an in-step co-ordination of work piece geometry, tools and peripherals, process programs and CAD data to meet high quality standards [8].

In iteratively performing these two functions for small steps of the total manufacturing process the benefits of the predictive planning of Virtual Prototyping can be used without making compromises in the process reliability and the product quality. The feedback incorporating 3D-sensor, robot and off-line programming is a possibility to meet the required high degree of flexibility in the production processes of the One-of-a-Kind products (Figure 10).

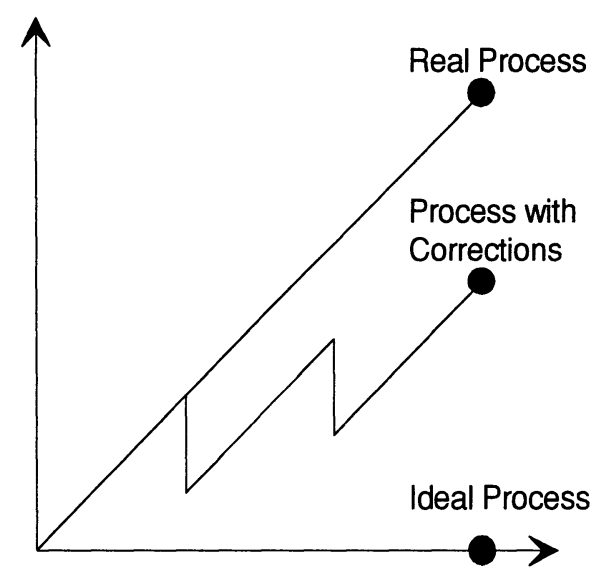

Figure 10 Tolerances of real, ideal and corrected processes

So One-of-a-Kind production can be automated, supplying repeatable results. By this, the tight time limits of prototype manufacturing can be held, and One-of-a-Kind products can be "right the first time", preserving from expensive and time-consuming corrections of defects. 


\subsubsection{Measurement System}

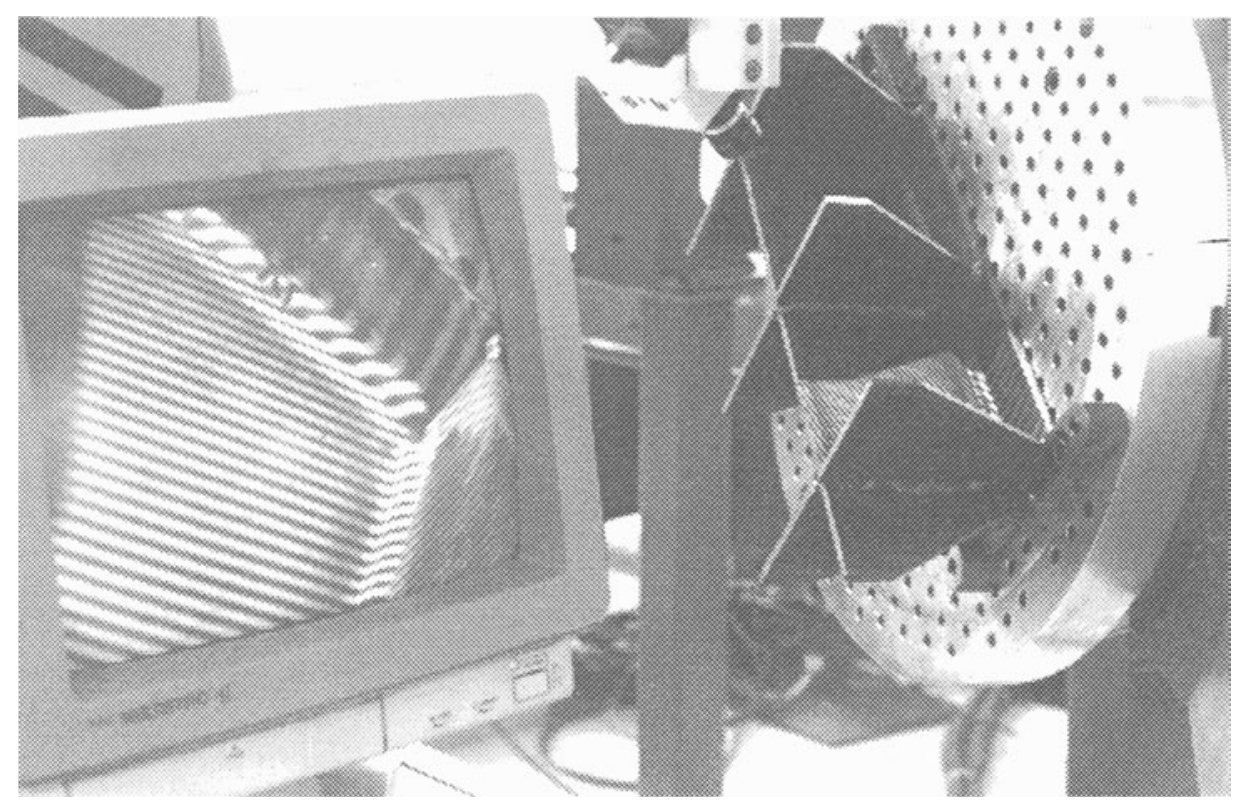

Figure 11 Steel construction part being measured by optical fringe projection system and coded light approach.

The 3D sensor utilises an active optical 3D-measurement process: the coded light approach. This is an absolute measurement method that requires only a small number of images to obtain a full depth-image within seconds. This is done by projecting sequences of line-patterns (Figure 11).

For the feed-back of geometric information acquired as 3D-clusters it is necessary to extract special points. First, collision spaces in the cell are examined. When there is no more risk of damage, the 3D-measurement tool and the CCD-cameras can be moved to the "points of interest". For many applications it is sufficient to search for corner points.

\subsection{Functionality of the Laboratory Robot Cell}

- cell layout design with IGRIP graphic robot simulation system,

- simulation of flame cutting, arc welding and assembly tasks for the CLOOS dual robot cell (13 axes),

- simulation of 3D-measurement with optical sensory system,

- translation of simulation programs and download of system control codes,

- acquisition of 3D-clusters with coded light approach,

- extraction of special points from a 3D-cluster,

- calibration of basic optical characteristics of 3D-measurement system,

- upload of system control codes and 3D-clusters,

- download of corrected system control codes. 


\subsection{Results}

In practice, the main needs that motivate the development of the described system are:

1. For the effective use of off-line programming, the layout data of the robot cell has to be updated by accounting the tolerance-caused offsets.

2. To supply for a high process reliability the robot's kinematic variations caused by payloads as well as the work piece tolerances have to be calibrated. The handling of flexible parts is also facilitated.

3. Faults and accumulated tolerances have to be detected in an early stage of the manufacturing process to minimise the costs and the delay for corrective actions. In addition the quality can be assessed in situ and can be documented.

4. When sequentially producing first a virtual prototype and then the real prototype the plan divergence and the process tolerances accumulate. This can be minimised by stepwise alternating between planning in the virtual environment and verification and calibration of the real environment.

\section{CONCLUDING REMARKS}

The ability to get a product out into market quickly is becoming the dominant requirement for manufacturing companies. This has to be realised with respect to an increasing demand for small quantities - in the extreme case of One-of-a-Kind products and a decreasing product lifeand innovation cycle. The motivation to be flexible and able to "make just what is needed when it is needed" is becoming stronger and stronger. The term "Rapid Response Manufacturing Systems" is used as proposed by Kohls and Moehring [9].)

"Concurrent Engineering (CE)" and "Integrated Product Development (IPD)" are generic terms for various approaches to allow designers and manufacturing engineers to communicate early in the design phase of a product. Multi-functional teaming is a means for increasing communication (and co-ordination), and has become the common approach towards improving responsiveness. The ability to do design iterations early in the product development cycle enhances design, improves quality and reduces costs [10]. The earlier that product improvements occur in the design process, the greater the savings will be. Beside various information management and groupware-tools, prototyping is a crucial concept to facilitate this early communication of ideas. Prototyping approaches range from soft prototypes, i.e. computer models of product or process design (Virtual Product Development, Virtual Manufacturing), to actual 3D physical engineering "hard" prototypes developed from CAD model data by a variety of Rapid Prototyping Technologies.

The modelling of process chains is an adequate approach to achieve better clarity of the product development. Process chains describe "what" has to be done and the information technological transactions between different activities. As well as for the description of the "As-Is" and the "Should-Be" situation, process chains have to be extended towards "How" it has to be done. The advantages of using this approach are the clarity and the possibility to choose and combine the most adequate activities aiming at a rapid response in product development.

Development of a generic reference model for rapid product development and One-of-a-Kind Production will provide an abstract view of the various process chains in a neutral and logical manner. In this sense the functions and relations of a reference model should be seen as a generic structure to derive the system architecture of product and part-specific future rapid response manufacturing process chains. 


\section{REFERENCES}

[1] Hirsch, B.E., Sheng, X.: Triangulation of trimmed surfaces in parametric, space computer-aided-design, Vol. 24, No. 8, August 1992.

[2] Mäntylä, M: An Introduction to Solid Modelling, Computer Science Press. USA, 1988.

[3] Berger, U., Thoben, K.-D., Müller, H.: Rapid Prototyping Technologies for Advanced Sheet Metal Forming. SME Rapid Prototyping \& Manufacturing '93 Conference, Dearborn, Michigan, USA, 1993.

[4] Müller, H., Berger, U., Thoben, K.-D.: Successful Application of Rapid Prototyping Technologies for Advanced Sheet Metal Forming and Investment Casting. Intelligent Manufacturing Systems, International Conference on Rapid Product Development, Stuttgart, Germany, 1994.

[5] König, W.: Fertigungsverfahren, Band 5: Blechumformung, Düsseldorf: VDI, 1990.

[6] N. N.: Company brochure of DENSIT ${ }^{\complement}$. Ålborg, Denmark.

[7] Berger, U., Schmidt, A., Wolf, H.: Optical system for robot based One-of-a-Kind manufacturing. EOS/SPIE European Symposium on Optics for Productivity and Manufacturing, Frankfurt/M., Germany, 1994.

[8] Barone, P. A.: "Techniques for Developing a Multi-Robot Cell for Production Processing of Complex Airframe Assemblies", 1989 SME Intern. Conf. and Exposition, pp. MS89399-1 to 28, Detroit, USA: SME, 1989.

[9] Kohls, J.B., Moehring, S.M.: Achieving Rapid Prototyping - Issues and Technologies to Facilitate Design and Prototyping, Third International Conference on Rapid Prototyping. Dayton, USA, 1992.

[10] Wozny, J.M.: "Research Trends in the U.S. for next generation CAD-Systems", CAD'92: Neue Konzepte zur Realisierung anwendungsorientierter CAD-Systeme. Berlin, Germany: Springer, 1992. 


\section{BIOGRAPHY}

Dr.-Ing. Klaus-Dieter THOBEN studied mechanical engineering at the TU Braunschweig. After finishing his studies, he was employed at the University of Bremen in the Faculty of Production Engineering. There, he received his doctor of engineering degree in CAD applications in 1989. In the same year he joined BIBA, where he led the CAD/CAM laboratory. Since 1991 he is head of the Department for Computer-Aided Design, Planning and Manufacturing. His special interests are computer-aided techniques and applications in product development, CIM and simultaneous engineering.

UIrich BERGER holds a degree of mechanical engineering from the University of Stuttgart and expects to finish his Ph.D. at the University of Bremen in May 1995. He is the technical manager of the BIBA institute. The professional experience of Mr. Berger contents responsible functions in the industrial automation and manufacturing industry.

Jürgen Friedrich BAUER graduated in Mechanical Engineering at University of Stuttgart in 1993. After a study project on Rapid Prototyping materials, he went to Daimler-Benz Research Centre in Ulm then, were he worked on 3D measurement systems for digitizing purposes in the context of the Intelligent Manufacturing Systems (IMS) initiative. Since 1994, he is scientist at BIBA Institute were he coordinates research activities in the field of Rapid Product Development and does technical project management in several EU funded RTD projects (Brite EuRam BE 5814 "Manufacturing Technologies for Sheet Aluminium Forming" (Hyprform), Brite EuRam BE 5278 "Optimisation of Rapid Prototyping Techniques for Automotive Industry").

Achim SCHMIDT graduated in Mechanical Engineering at University of Stuttgart. From 1983 to 1993, he was research assistant at Fraunhofer Institutes (IPA, IBP) and the University Computer Centre at Stuttgart for data processing, software development, graphic simulation and robot control. Since 1993 he is research scientist at BIBA in the fields of robotics, off-line simulation, virtual environments, autonomous systems and optical sensors. Since 1994, Mr. Schmidt is involved in the ESPRIT project No 8338: NEUROBOT - Neural Network-Based Industrial Robots for Disassembly and Recycling of Automotive Products. 